\title{
A Formalised Ontology of Musical Instruments
}

\author{
Ibiyinka Temilola Ayorinde \\ Department of Computer Science \\ University of Ibadan, \\ Ibadan, Nigeria
}

\begin{abstract}
A formal ontology is an aspect of knowledge representation that deals with the conceptualization of domains. As ontology enhances the sharing of common understanding of the structure of information, it can be used as a training tool. Hence, this article develops a formalised ontology of musical instruments that is based on the Hornbostel and Sach's classification scheme. The ontology provides information about the families, groups and characteristics of musical instruments such as the shape and how it is played, among others. It also recommends what instrument to learn based on the user's preference. The concepts of the ontology and the relationship between them are formalised using the predicate logic and implemented with Prolog programming language.
\end{abstract}

\section{Keywords}

Formal Ontology, Musical instruments, Training tool, Prolog, Predicate logic.

\section{INTRODUCTION}

An ontology is usually focused on an explicit specification of conceptualisation of the objects, concepts, and entities that are presumed to exist in certain area of interest and the relationships that hold among them. It has the meaning of a standardized terminological framework in terms of which the information is organized $[1,2,3]$. Ontologies ensure efficient retrieval by enabling inferences based on domain knowledge, which is gathered during the construction of the knowledge base [4]. It also helps to spell out the facts in a domain and hence, creates rules and constraints that bind the relationships between the concepts together $[5,6,7]$. Formalized ontology constructs a formal codification for the knowledge elicitation of the concerned domain [1].

Music is a collection of coordinated sound or sounds [8]. It brings about melody and hence helps brings positive effects on health by improving mood, reducing stress, lessoning anxiety and improving memory among others [9]. Musical instruments are used to make musical sounds. Musical instruments evolved in step with changing applications and technologies hence, different musical instruments have evolved over the years [10]. Musical ontology is the study of the kinds of musical things that exist and the relations that hold between them [11]. The ontology developed in this study is based on the Hornbostel and Sach's classification scheme [12]. Since reasoning aims at extracting information not directly represented and ontologies can be complemented with reasoning capabilities, mainly through the application of rules on the given facts, prolog programming language is chosen to implement this ontology [13, 14].

\section{RELATED WORK}

\subsection{Knowledge Representation Issues In Musical Instrument Ontology Design:}

This paper presents preliminary work on musical instruments ontology design, and investigates heterogeneity and limitations in existing instrument classification schemes. The authors developed representations using the Ontology Web Language (OWL), and compared terminological and conceptual heterogeneity using SPARQL queries. They found evidence to support that traditional designs based on taxonomy trees lead to ill-defined knowledge representation, especially in the context of an ontology for the Semantic Web. In order to overcome this issue, they suggested an instrument ontology that exhibits a semantically rich structure [15].

\subsection{The Music Ontology:}

This work develops a distributed music information system that aims at gathering music related information held by multiple databases or applications. Semantic Web technologies were used to create a unified information environment. The author developed a formal ontology for the music domain which allows a published and interlinked a wide range of structured music-related data on the Web. Different technologies were also developed along with an algorithm that automatically relate music-related datasets among each other. Three of the applications developed were described using the distributed information environment [16].

\subsection{Towards the Automatic Generation of A Semantic Web Ontology for Musical Instruments:}

This article presents a novel hybrid ontology generation system for musical instruments. The work focuses on automatic instrument taxonomy generation in Ontology Web Language (OWL). The hybrid system consists of three main units which are musical instrument analysis, Formal Concept Analysis and lattice pruning and hierarchical form generation [17].

\section{METHODOLOGY}

\subsection{The Ontology Template}

The conceptual modelling of the musical instruments developed in this study involves defining classes in the ontology, arranging the classes in a taxonomic hierarchy, defining slots and describing allowed values for these slots and filling in the values for slots for instances and modeling the ontology. This is followed by writing the axioms in predicate logic for the various facts and rules in the ontology. The predicate logic axioms were translated into prolog axioms and competency questions were used to draw inferences from the ontology. There are 4 main classes defined in this ontology. They are: Chordophones, Aerophones, Percussion and Keyboard. The class hierarchy is shown in Fig. 1. 


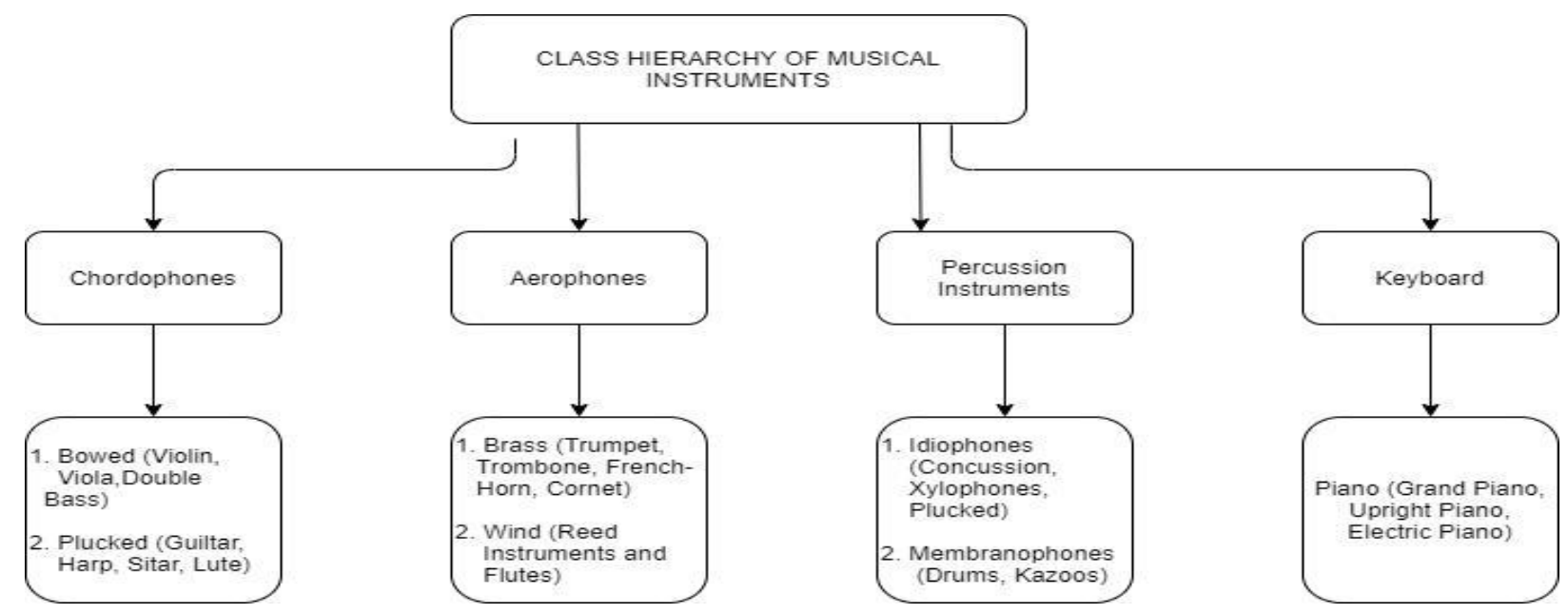

Fig. 1: Class Hierarchy of the Musical Instruments

Some of the properties used are: isPlayed, isBowed, isPlucked, is_A, isWooden, isMetal, familyOf, hasPegs, hasBow, hasStrings, isClapped, typeOf, plays-with and shapeOf among others. A few of the facts and rules in the ontology are given below:

i. Instruments can be in different families of Chordophones, Aerophones, Percussion and Keyboard.

ii. A string instrument can be bowed or plucked.

iii. An instrument is a string instrument if it has strings, pegs and is wooden.

iv. A plucked string instrument is usually played by plucking.

v. Guitar is a plucked instrument.

vi. A violin has pegs, strings and is wooden.

vii. A violin is played by bowing

\subsection{Axioms}

Some of the axioms written in predicate logic are shown below:

Axiom 1: The musical instruments are classified into Chordophones, Aerophones, Percussion and Keyboard.

$\forall \mathrm{x}, \quad$ MusicalInstrument(x) $\leftrightarrow \quad$ Chordophones(x) $\quad \vee$ Aerophones $(\mathrm{x}) \vee \operatorname{Percussion}(\mathrm{x}) \vee \operatorname{Keyboard}(\mathrm{x})$

Axiom 2: Chordophones can either be bowed or plucked_C instruments

$\forall \mathrm{x}$, Chordophones(x) $\leftrightarrow$ Bowed(x) $\vee$ Plucked_C(x)

Axiom 3: Aerophones is divided into Brass, Wind or Flutes

$\forall \mathrm{x}$, Aerophones (x) $\leftrightarrow \operatorname{Brass}(\mathrm{x}) \vee \operatorname{Wind}(\mathrm{x}) \vee$ Flute( $\mathrm{x}$ )

Axiom 4: Percussion is divided into Idiophones and Memranophones

$\forall \mathrm{x}$, Percussion $(\mathrm{x}) \leftrightarrow$ Idiophones(x) $\vee$ Memranophones(x)

Axiom 5: Idiophones can be Concussion, Xylophones, Plucked_I or Shaken

$\forall \mathrm{x}$, Idiophones $(\mathrm{x}) \leftrightarrow$ Concussion $(\mathrm{x}) \vee$ Xylophones $(\mathrm{x}) \vee$ Plucked_I $(\mathrm{x}) \vee$ Shaken $(\mathrm{x})$

Axiom 6: A Chordophones has pegs, strings and is wooden. $\forall \mathrm{x}$, Chordophones $(\mathrm{x}) \leftrightarrow \operatorname{hasPegs}(\mathrm{x}) \wedge \operatorname{hasStrings}(\mathrm{x}) \wedge$ isWooden $(\mathrm{x})$

Axiom 6: A Violin is an instance of Chordophones

$\forall \mathrm{x}, \operatorname{Violin}(\mathrm{x}) \rightarrow \operatorname{hasPegs}(\mathrm{x}) \wedge \operatorname{hasStrings}(\mathrm{x}) \wedge \operatorname{isWooden}(\mathrm{x})$

Axiom 7: Every concussion instrument is clapped

$\forall \mathrm{x}, \operatorname{classOf}(\mathrm{x}$, concussion $) \leftrightarrow$ isClapped(x).

Axiom 8: Chordophones are string instruments

$\forall \mathrm{x}$, familyOf(x, string) $\leftrightarrow$ Chordophones(x)

Axiom 9: Aerophones are wind instruments

$\forall \mathrm{x}$, familyOf(x, string) $\leftrightarrow$ Aerophones(x)

Axiom 10: An instrument can be played with hand if it is a family of strings, percussion or keyboard.

played_with(x,hand) $\leftrightarrow$ familyOf(x, strings) $\vee$ familyOf(x, percussion $) \vee$ familyOf(x, keyboard).

Axiom 11: An instrument can be played with the mouth if it is a family of wind

played_with(x,mouth) $\leftrightarrow$ familyOf(x, wind).

\subsection{Competency Questions}

Some of the questions used to test the scope and competency of the ontology are given below:

1. What can you learn in this ontology?

2. What are the musical instruments that exist in this ontology?

3. What musical instruments belong to the string family?

4. Which family does the trumpet belong to?

5. What are the available wind instruments?

6. What instrument do I learn if I want to play songs of blues genre?

\section{IMPLEMENTATION AND RESULTS}

All the predicate logic axioms were translated into prolog axioms. SWI (Sociaal-Wetenschappelijke Informatica ("Social Science Informatics")) Prolog was used to implement this ontology. The predicates denote the relations in the domain. The following Fig.s show the results for some queries. The upper part of the SWI prolog window is the 
prolog editor while the lower part of the window is where the queries are answered.

\subsection{Result of the Query: start.}

Fig. 2 shows the result of the query "start". Six options are shown here and the user can pick any of the options depending on what he/she wants to know about the ontology.

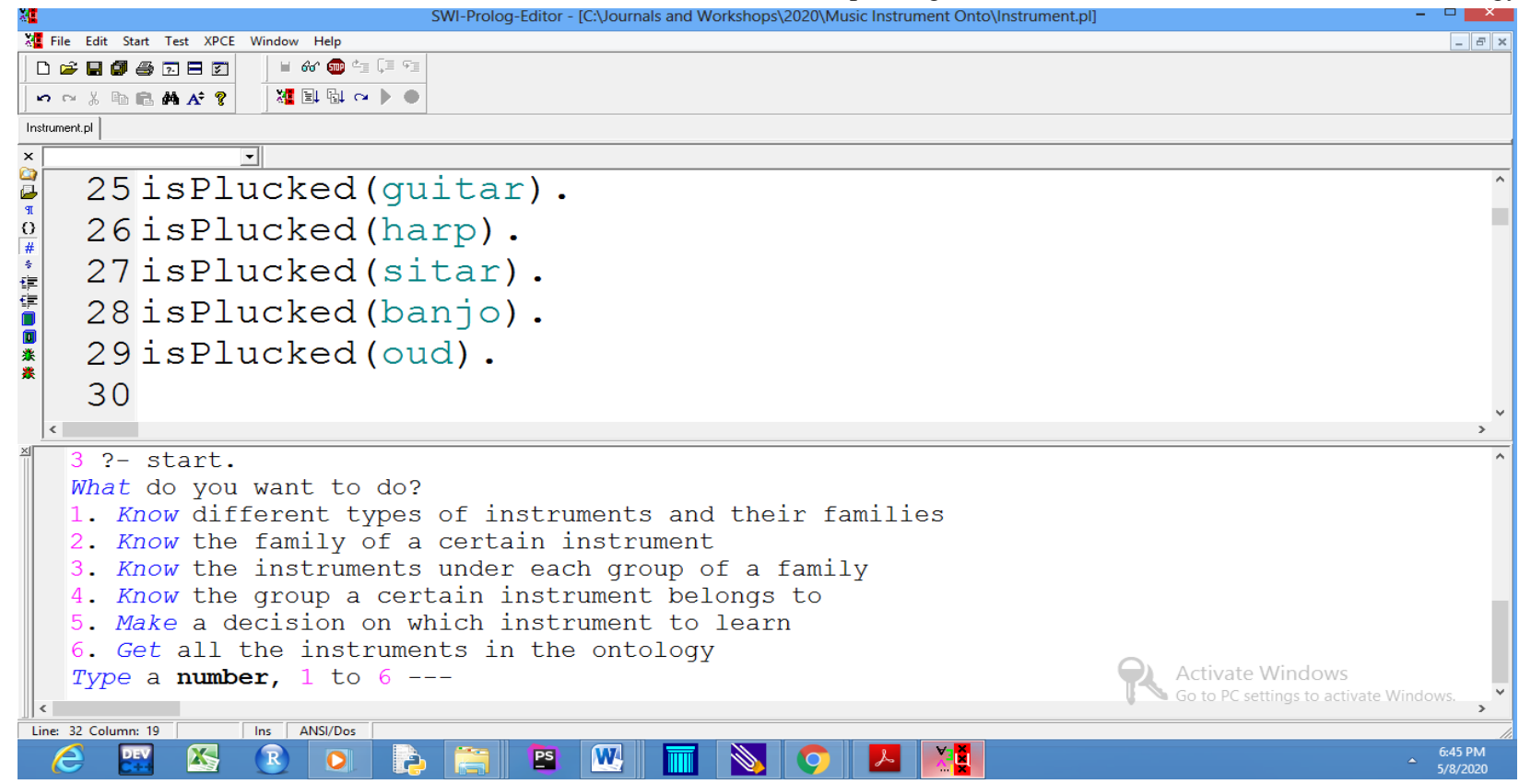

Fig. 2: Answer to the Query: "Start:

The six options are:

1. Know different types of instruments and their families

2. Know the family of a certain instrument

3. Know the instruments under each group of a family

4. Know the group a certain instrument belongs to

5. Make a decision on which instrument to learn

6. Get all the instruments in the ontology
The learner can choose what to learn or know from the six options.

\subsection{Result for the Query: "Which instruments belong to the string family?"}

Fig. 3 shows the instruments that belong to the family of string instruments. Some of the instruments shown in the result are: violin , viola, doublebass, cello, guitar, harp, sitar, banjo, oud, violin and bassguitar among others.

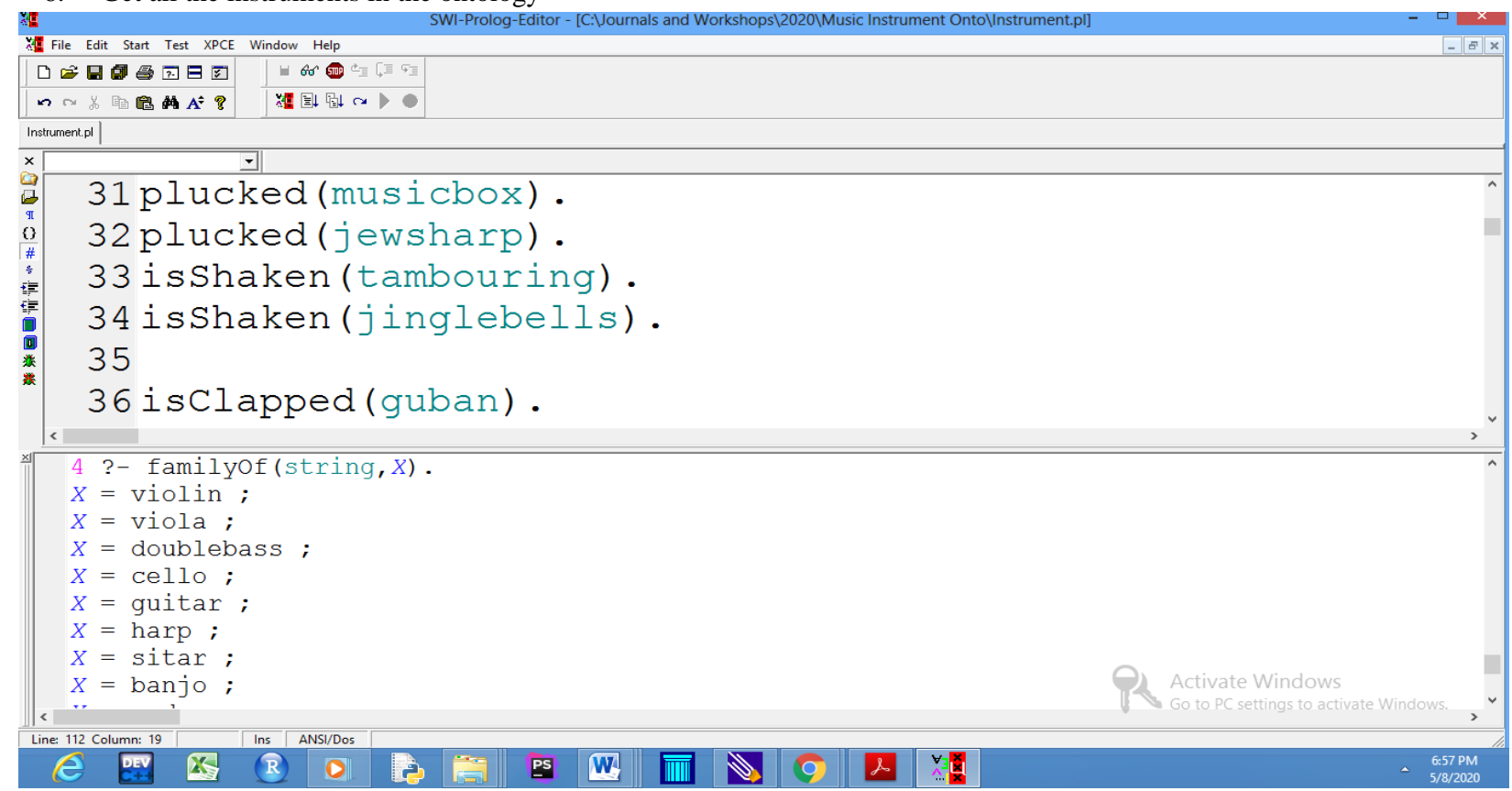

Fig. 3: Answer to the Query: "Which instruments belong to the string family?" 


\subsection{Answer to the Query: "What are the instruments that exist in this ontology?"}

Fig. 4 shows the answer to the instruments that exist in the ontology. Only part of the instruments could be shown as the list is longer than the interface.

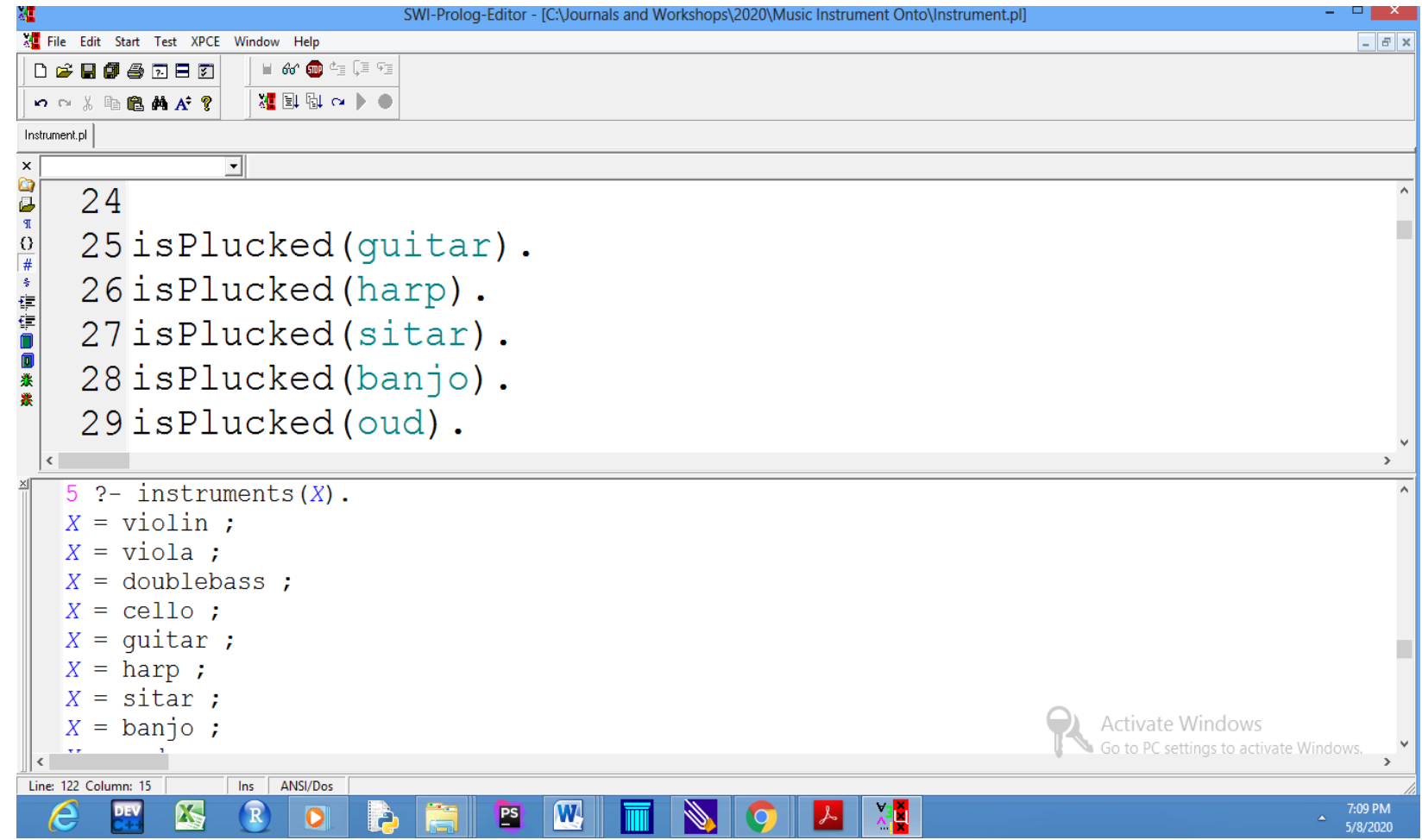

Fig. 4: Answer to the query: "What are the musical instruments that exist in this ontology?"

Some of the answers given are violin, viola, doublebass, cello, guitar, harp, sitar, banjo, oud, bassguitar, violin, viola, flutemain, clarinet, altosax, sopranosax, tenorsax, oboe, basson, harmonica, reed_organ, trumpet, trombone, frenchhorn and cornet among others.

\subsection{Result of the Query: "Which family does the trumpet belong to?"}

Fig. 5 shows the answer to the query requesting for the family of the trumpet as "Wind".

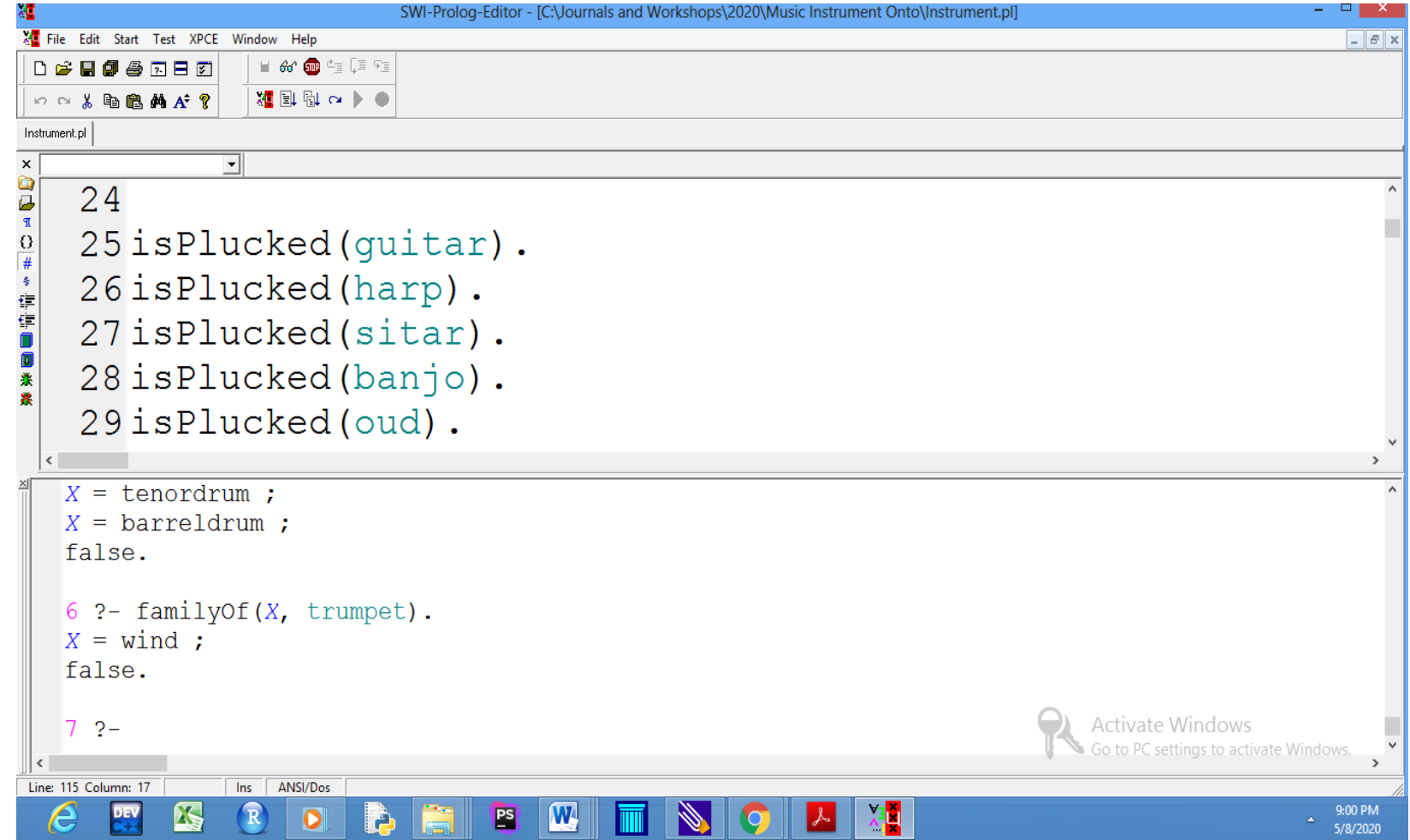

Fig. 5: Answer to the query: "Which family does the trumpet belong to?" 


\section{CONCLUSION}

The musical instrument ontology developed in this study gives a good classification and characteristics of the wind (also known as aerophones), string (also known as chordophone), percussions and keyboard. It can be used as a learning tool for beginners who want to start learning music or musical instruments.

\section{REFERENCES}

[1] Corazzon, R. (2019). Theory and History of Ontology.

[2] Gruber, T. R. (1993). A Translation Approach to Portable Ontology Specifications. Knowledge Acquisition 5 (2): 199-220

[3] Panov, P., Soldatora L. N. and Dzeroski S. (2009). Towards an Ontology of Data Mining Investigations. J. Gama et al. (Eds). In Discovery Science: 12th International Conference, DS 2009, Porto, Portugal . LNAI 5808, pp 257 - 271.

[4] Deshpande, N. J. and Kumbhar, R. (2011). Construction and Applications of Ontology: Recent Trends. DESIDOC Journal of Library \& Information Technology, Vol. 31, No. 2, March 2011, pp. 84-89

[5] Neuhaus, F. (2017). On the Definition of 'Ontology' Proceedings of the conference on The Joint Ontology Workshops (JOWO), September, 2017 at : Bolzano

[6] Ayorinde, I. T. \& Oyedeji, O. A. (2019). An Ontology for Intra-Campus Transport System (ICTS) (A Case Study of the University of Ibadan Campus). Journal of Digital Inovations and Contemp. Res. In Sc., Eng., \& Tech.. Vol. 7. No. 4: 65-78.

[7] Guarino, N. and Giaretta, P. (1995). Ontologies and knowledge bases: In book: Towards VeryLarge Knowledge Bases. Publisher: Amsterdam:IOS Press. Editors: Mars, N.J.I.

[8] What is Music? - Definition, Terminology \& Characteristics. (2019, March 19). Retrieved from https://study.com/academy/lesson/what-is-musicdefinition-terminology-characteristics.html.

[9] Pfizer Medical Team. (2017). 10 Health Benefits of Music. Published on Aug 30, 2017https://www.gethealthystayhealthy.com/articles/10health-benefits-of-music. Retrieved 12-4-2020

[10] Montagu, J. (2007), Origins and Development of Musical Instruments, The Scarecrow Press, ISBN 978-0-8108$5657-8$

[11] Stanford Encyclopedia of Philosophy (2017). The Philosophy of Music

[12] Lee, D. (2019). Encyclopedia of Knowledge Organization. Edited by Hjørland B. and Gnoli C. https://www.isko.org/cyclo/hornbostel

[13] Horrocks, I., Patel-Schneider, P. F., Boley, H., Tabet, S., Grosof, B. and Dean, M.

[14] (2004)SWRL: A Semantic Web Rule Language Combining OWL and RuleML. W3C Member Submission 21 May 2004.

[15] Motik, B., Sattler, U. and Studer, U. (2004). Query answering for OWL-DL with rules. In Proceedings of the Third International Semantic Web Conference (ISWC 2004), Hiroshima, Japan, Nov 2004, pp. 549 563.

[16] Kolozali, S., Barthet, M., Fazekas, G. and Sandler, M. (2011). Knowledge Representation Issues In Musical Instrument Ontology Design.

[17] Raimond, Y. (2008). A Distributed Music Information System. PhD thesis, Queen Mary University of London.

[18] Kolozali, S., Barthet, M., Fazekas, G. and Sandler, M. (2010). Towards The Automatic Generation of A Semantic Web Ontology For Musical Instruments. International Conference on Semantic and Digital Media Technologies. SAMT 2010: Semantic Multimedia pp 186-187. 